\title{
Assessment of natural variations of key fossil parameters in conifer species and their impact on the accuracy of the leaf gas exchange model for ancient $\mathrm{CO}_{2}$ reconstruction
}

\author{
JIAQI LIANG ${ }^{1}$, QIN LENG ${ }^{1}$, DAIANNE HÖFIG ${ }^{2}$, GAO \\ $\mathrm{NIU}^{1}$, LIANG XIAO $^{3}$, YI GE ZHANG ${ }^{2}$ AND HONG YANG ${ }^{1}$

The leaf gas exchange model (i.e., the Franks Model) is increasingly applied to reconstructing ancient atmospheric $\mathrm{CO}_{2}$ levels due to its ability to produce well-constrained $\mathrm{CO}_{2}$ estimates at low or high levels and is not restricted to fossil taxa with modern relatives. The model requires input parameters from plant fossils: bulk leaf carbon isotopic composition $\left(\delta^{13} \mathrm{C}\right)$, whole leaf stomatal density, and stomatal measurements such as guard cell width, guard cell length, and pore length. The natural variations of these parameters have not been well assessed, hindering our understanding of their impact on model results. We evaluated these variations and model their impact on the Franks Model output using extinct and extant species of three conifers, Metasequoia, Sequoia, and Taxodium, which are commonly used for ancient $\mathrm{CO}_{2}$ reconstruction.

We demonstrated statistically that key stomatal parameters are the least variable in the middle portion of a leaf needle from the middle part of a conifer branchlet. This observation supports the traditional notion that such samples provide consistent cuticular characteristics in palaeobotanical research. Among the three measurable stomatal parameters from fossils, the pore length has the largest variability, while guard cell width and length are relatively stable. In contrast, we found little variation in $\delta^{13} \mathrm{C}$ values at different parts of a conifer leaf as well as from eaves at various locations along a branchlet. Modeling tests show that pore length and whole leaf stomatal density are high impact parameters due to a combination of high model sensitivity and a large range of natural variations. Our new data have several implications for enhancing the accuracy of the gas exchange model. With an improved cleared leaf preparation for precise measurements of key stomatal parameters, a statistically informed counting strategy, and a careful selection of scaling factors will expand our ability to better reconstruct ancient $\mathrm{CO}_{2}$ concentrations through geological time using these conifer genera. 